\title{
Pharmacotherapy for Neurocognitive Disorders Based on the Hypothesis of Endogenous Appearance of Anticholinergic Activity
}

\author{
Misa Hosoi ${ }^{a}$ Koji Horia Kimiko Konishia,b Masayuki Tanic Hiroi Tomioka ${ }^{a}$ \\ Yuka Kitajimad $^{d}$ Norihisa Akashie Atsuko Inamoto $^{a}$ Kenzo Kurosawa ${ }^{a}$ \\ Hajime Yuda $^{a}$ Takahiro Hanashi $^{a}$ Hiromi Ouchi ${ }^{a}$ Mitsugu Hachisu ${ }^{f}$ \\ a Department of Psychiatry, Showa University Northern Yokohama Hospital, Yokohama, and b Tokyo Metropolitan \\ Tobu Medical Center for Persons with Developmental/Multiple Disabilities, ${ }^{C}$ Department of Psychiatry, \\ Showa University East Hospital, 'Department of Anesthesiology, School of Medicine, Juntendo University, \\ e Department of Psychiatry, Showa University Karasuyama Hospital, and f Department of Pharmaceutical \\ Therapeutics, Division of Clinical Pharmacy, School of Pharmacy, Showa University, Tokyo, Japan
}

\section{Key Words}

Neurocognitive disorders · Serum anticholinergic activity · Acetylcholine $\cdot$ Cholinesterase inhibitor .

$\mathrm{N}$-methyl-D-aspartate receptor antagonist

\begin{abstract}
We previously proposed the hypothesis of endogenous anticholinergic activity $(A A)$ in Alzheimer's disease (AD). According to this hypothesis, the downregulation of acetylcholine seen in $A D$ is associated with upregulation/hyperactivity of N-methyl-D-aspartate receptor (NMDAR). The hyperactivation of NMDAR then induces inflammation, which, in turn, causes AA to appear endogenously. Based on this hypothesis, we commented that cholinesterase inhibitors (ChEls) are 'preventative' therapy for AD and NMDAR antagonists are the true 'treatment' for AD. We also noted that ChEls, such as donepezil, could treat delirium. Moreover, we proposed measuring serum anticholinergic activity in patients, particularly AD patients, in out-of-hospital pharmacies to monitor the anticholinergic burden for targeted treatment.
\end{abstract}

(c) 2015 S. Karger AG, Basel
(C) 2015 S. Karger AG, Basel

$1660-2854 / 15 / 0153-0157 \$ 39.50 / 0$

\section{Introduction}

In Japan, donepezil was approved for treating Alzheimer's disease (AD) in 1999, followed by galantamine, rivastigmine and memantine in 2011. Moreover, donepezil was approved for treating Lewy body disease (LBD) in 2014. Donepezil, galantamine and rivastigmine are cholinesterase inhibitors (ChEIs); these medications are used because downregulation of acetylcholine (ACh) has been implicated in AD [1]. Memantine is an N-methyl-D-aspartate receptor (NMDAR) antagonist (NMDAR-A), used because NMDARs have also been implicated to be hyperactivated in $\mathrm{AD}[2]$.

ACh controls not only cognitive function, but also inflammatory activity in both the central nervous system and peripheral tissues. In $\mathrm{AD}$, the cholinergic system is downregulated [1]; thus, inflammatory processes in both the central nervous system and peripheral tissues may be caused by downregulation of ACh [3]. NMDAR expression is also upregulated in central nervous system disorders, leading to hyperactivity of the inflammatory system

\section{KARGER 125}

E-Mail karger@karger.com

www.karger.com/ndd
Koji Hori, MD, PhD

Department of Psychiatry, Showa University Northern Yokohama Hospital 35-1 Chigasakichuo, Tsuzukiku

Yokohama City, Kanagawa 224-8503 (Japan)

E-Mail kojihori@med.showa-u.ac.jp 
[4]. Cytokines that have anticholinergic activity (AA) may appear as a result of this inflammation [5]. Therefore, we previously hypothesized that both AA in the central nervous system and peripheral tissue (serum anticholinergic activity, SAA) may appear endogenously in the moderate stage of $\mathrm{AD}$ [6].

Moreover, muscarinic- 1 receptor agonists induce amyloid precursor proteins to become nonamyloid proteins ( $\alpha$-processing) [7]. Therefore, muscarinic receptor antagonism (i.e. AA) favors the conversion of amyloid precursor proteins to amyloid, worsens cognitive function and exacerbates $\mathrm{AD}$ pathology by increasing amyloid plaques. Based on this speculation, we consider that downregulation of ACh and amyloid pathology interact in a synergistic manner.

In this article, we propose a better-targeted pharmacotherapy for $\mathrm{AD}[8]$ according to the AA hypothesis [9$11]$. We also recommend monitoring patients' SAA in out-of-hospital pharmacies as a guide to disease progression and pharmacotherapy.

\section{Pharmacotherapy Based on the Anticholinergic Hypothesis}

We speculate that 3 amyloidogenic patterns appear in $\mathrm{AD}$ [11]. The first is the physiological pattern ( $\mathrm{N}$ pattern), which is related to the normal aging process. We speculate that the $\mathrm{N}$ pattern of amyloidosis may be necessary for normal presenile or senile brain maturation. The second is the pathological pattern (P1), which is unrelated to the ACh downregulation observed in mild cognitive impairment (MCI) or mild AD. The third is also a pathological pattern (P2) and is related to the ACh downregulation observed in moderate $\mathrm{AD}$. While the $\mathrm{N}$ pattern may begin during normal aging, the $\mathrm{P} 1$ pattern probably begins when the clinical symptoms of mild AD occur. P1-pattern amyloid is likely to be misdiagnosed as the normal aging pattern because of the slowness of cognitive decline. However, the $\mathrm{P} 2$ pattern is clearly prominent and readily diagnosed as moderate $\mathrm{AD}$ when clinical symptoms such as memory disturbance, disorientation, aphasia, delusions, hallucinations and diurnal rhythm disturbance are present. In the moderate stage, the rate of cognitive decline is also more rapid than that in MCI or mild AD [11].

Based on this hypothesis, we believe that pharmacological agents for treating dementia (i.e. ChEIs and NMDAR-As) are partially symptomatic treatment (for P1-pattern amyloid) and partially disease-modifying treatment (for P2-pattern amyloid). We also speculate that true 'treatment' for $\mathrm{AD}$ begins in the moderate stage using NMDAR-As and that 'prevention' by prescribing ChEIs is in order to 'prevent' the appearance of AA [8].

From these speculations, we propose pharmacotherapies for $\mathrm{AD}$ as follows. If the progression of $\mathrm{AD}$ has been slow throughout, there would be no necessity for medical treatment. In contrast, when $\mathrm{AD}$ reaches a moderate stage, it progresses more rapidly than before. Therefore, ChEIs are necessary to delay the appearance of this rapid decline, and NMDAR-As are necessary to decrease the rate of this decline. If the pharmacotherapies work well, the progression of $\mathrm{AD}$ is slow [8].

This AA hypothesis can also explain 2 other facts. First, the experimental amyloid vaccine tested in animal models of $\mathrm{AD}$ has met with only limited success. If $\mathrm{N}$ pattern amyloid is necessary for normal brain maturation, only P1- and/or P2-pattern amyloid should be abolished. However, the proposed amyloid vaccine would abolish all 3 types. Second, ChEIs do not prevent conversions from MCI to mild dementia. At present, there is no preventative therapy or effective pharmacotherapy for amyloidogenesis of the $\mathrm{P} 1$ pattern [11]. We consider that because amyloid pathology is evident even in the MCI stage, ChEIs should be prescribed. Although ChEIs cannot prevent the conversion from MCI to mild dementia, this medication can delay the conversion from mild dementia to moderate dementia.

Five milligrams of the medication donepezil are allowed for mild-to-moderate stages of AD after 1-2 weeks of 3-mg dosing, and $10 \mathrm{mg}$ of donepezil is allowed daily for the severe stage of AD. However, in LBD, $10 \mathrm{mg}$ of this medication should be prescribed daily after upward titration from $3 \mathrm{mg}$ for 1-2 weeks and $5 \mathrm{mg}$ for 4 weeks. This medication regimen has been recommended considering that downregulation of $\mathrm{ACh}$ is believed to be severer in $\mathrm{LBD}$ than in $\mathrm{AD}$ [12]. We believe this, too. However, because of patient fragility during treatment with antipsychotic agents in LBD [13], even ChEIs should be prescribed for LBD prudently.

Recently, Yilmaz et al. [14] presented a report where they describe the case of a 19-year-old male who was brought to the emergency room by his relatives for initial complaints of restlessness. He was later diagnosed with poisoning by Datura stramonium, a plant widespread in Turkey as herbal wealth and containing atropine (anticholinergic medicine); hence, this was a case of anticholinergic toxicity. He had ingested D. stramonium $4 \mathrm{~h}$ before developing symptoms. When he was admitted, he showed restlessness and disorientation to time and place. Ten milligrams of midazolam were administered for agi- 
Fig. 1. We speculate that ACh downregulation not only causes cognitive dysfunction and behavioral and psychological symptoms of dementia (BPSD), but also induces inflammation in the central and peripheral nervous systems. This induces AA both centrally and peripherally through cytokine pathways. Moreover, AA causes an increase in amyloid and further decreases $\mathrm{ACh}$. This is referred to as the 'endogenous AA cascade'. This figure is reproduced from an article by Hori et al. [9]. The procedure was permitted by the Japanese Society of Neuropsychopharmacology (Tokyo, Japan).

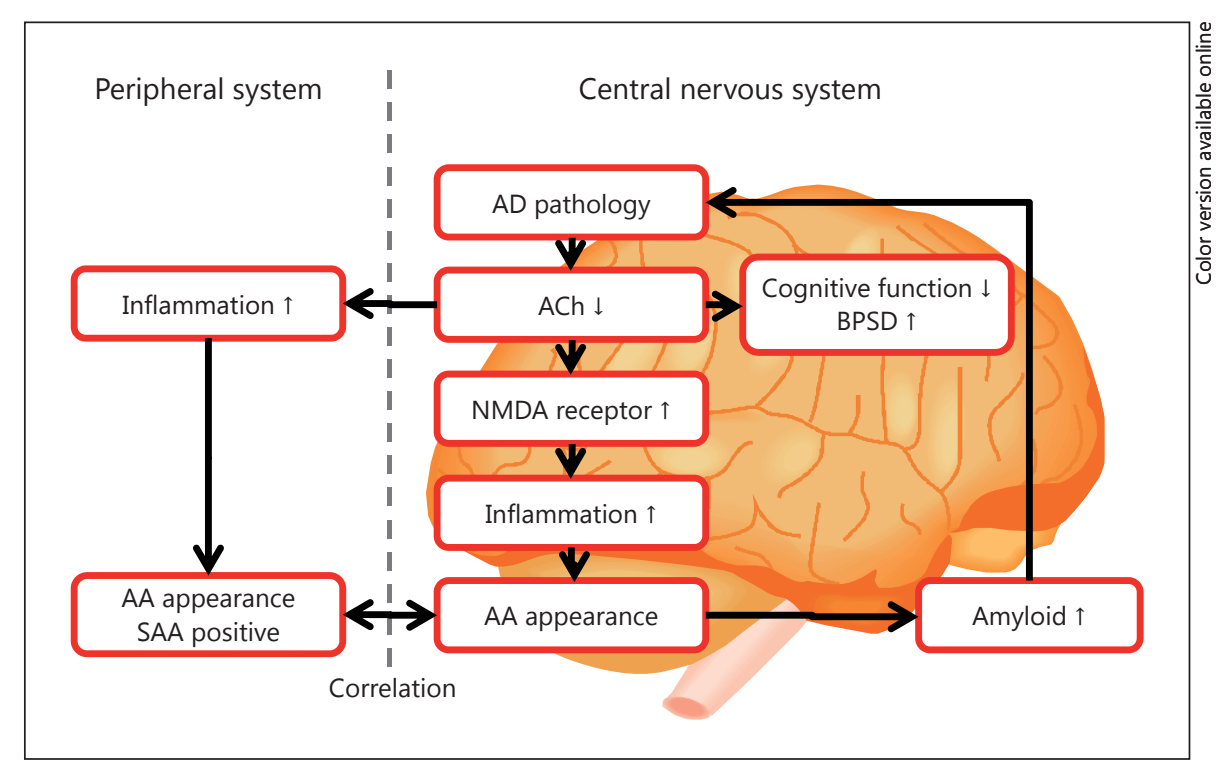

tation. Agitation ceased but then recurred. Two milligrams of physostigmine were administered, which caused sudden recovery from the symptoms. From this case report, we also consider that this patient's cholinergic system was not fully mature as they commented; therefore, the heightened AA allowed toxicity to occur. We are convinced of the existence of an 'endogenous anticholinergic cascade' (fig. 1; courtesy of Hori et al. [9]).

We speculate that D. stramonium caused a heavy anticholinergic burden; however, because it lasted for a long time, appearance of endogenous AA should also be considered. As such an endogenous AA appearance system, we previously proposed the 'endogenous anticholinergic cascade' (fig. 1; courtesy of Hori et al. [9]). Because the anticholinergic toxicity disappeared suddenly after administering a single dose of physostigmine, we also speculate that upregulation of ACh acts not only to antagonize $\mathrm{AA}$ at the ACh receptor, but also to abolish AA directly. We previously reported on a patient with long-term alcohol-related delirium that was ameliorated by the ChEI donepezil [15]; in another patient with MCI or mild AD, AA disappeared with donepezil treatment [16]. These patients showed the importance of upregulation of ACh. The case report by Yilmaz et al. [14] convinces us of the existence of an 'endogenous anticholinergic cascade' [9], even though the origin of AA is related with exogenous factors. We are also convinced that upregulation of $\mathrm{ACh}$ abolishes AA directly in delirium in other clinical settings. Therefore, we consider that ChEIs such as donepezil have the ability to treat delirium.

Pharmacotherapy for Neurocognitive Disorders Based on AA

\section{Proposal to Measure SAA in Out-of-Hospital Pharmacies}

Endogenous production is not the only cause of elevated AA; rather, prescribed medication is the main reason for it [17]. We propose establishing a medicationmonitoring system in out-of-hospital pharmacies. In Japan, a patient may receive multiple prescriptions from more than 1 hospital. Therefore, this system causes physicians to not know the true pharmacological regimen of all their patients. Because no one knows about the other prescriptions, polypharmacy may occur. Polypharmacy is a risk factor for delirium [17] because of inadvertent anticholinergic toxicity. This can cause various side effects, such as cognitive dysfunction, psychotic symptoms and progression of dementia, as we have reported previously $[18,19]$. We have proposed to measure SAA [20] in out-of-hospital pharmacies. If SAA is positive, a pharmacist asks the involved physician to perform a pharmaceutical review and reduce or change the pharmacological regimen of the patient. If the number of medicines being taken decreases, the patient's anticholinergic burden is likely to decrease. Accordingly, adverse effects caused by AA should decrease, including the progression of dementia as per our endogenous hypothesis of $\mathrm{AA}$ in $\mathrm{AD}$. We have previously reported the usefulness of measuring SAA to evaluate anticholinergic toxicity in a dementia patient [21]. We showed the example of this system in figure 2. If patient A was prescribed 4 medicines in hospital 1, 3 in hospital 2, 3 in 


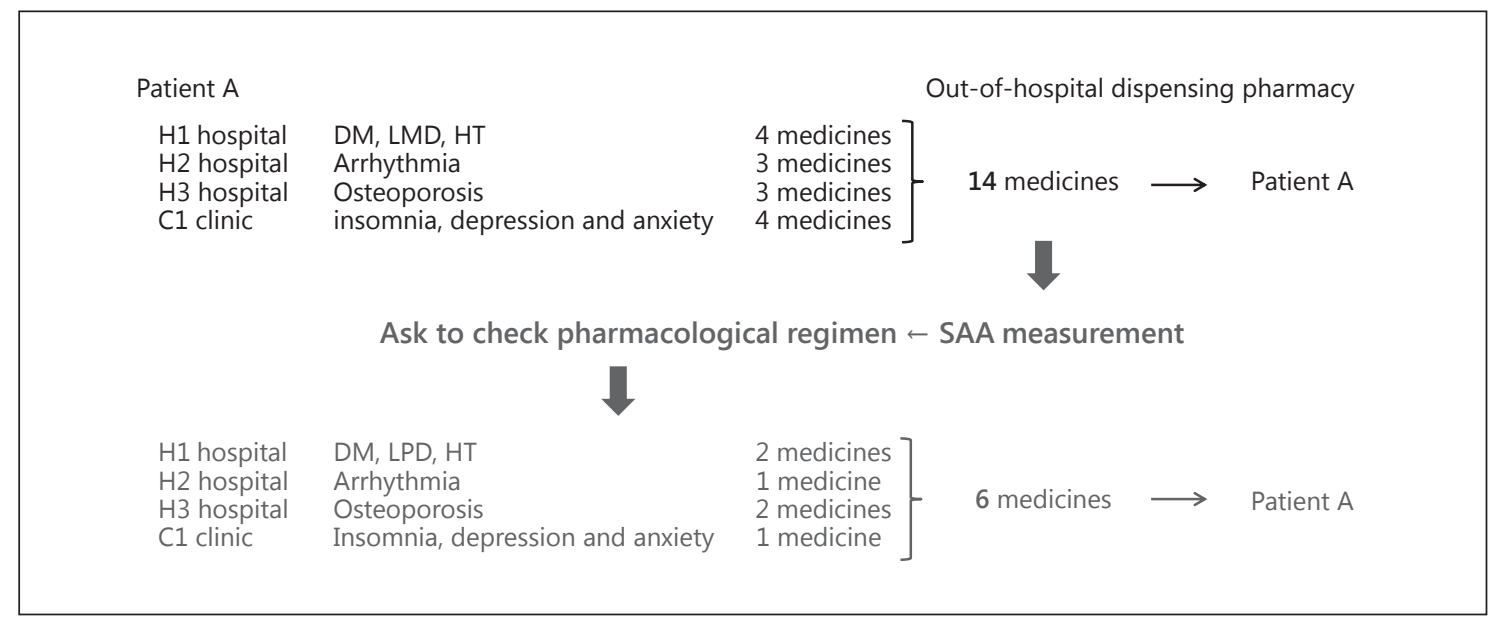

Fig. 2. If patient A were prescribed 4 medicines in hospital 1, 3 in hospital 2, 3 in hospital 3 and 4 in clinic 1, he would receive a total of 14 medicines. If SAA were measured and found to be positive, the pharmacists could ask each physician to review and reduce or change the patient's pharmacological regimen. If the number of medicines taken decreased, the patient's anticholinergic burden would possibly decrease. DM = Diabetes mellitus; LMD = lipid metabolic disorder; HT = hypertension.

hospital 3 and 4 in clinic 1 , he would receive a total of 14 medicines. If SAA was measured and found to be positive, the pharmacists could ask each physician to review and reduce or change the patient's pharmacological regimen. If the number of medicines taken decreases, the patient's anticholinergic burden would possibly decrease.

\section{Conclusion}

We have commented on the pharmacotherapy of dementia according to our hypothesis of endogenous AA in $\mathrm{AD}$. We speculate that antidementia agents such as ChEIs and NMDAR-As are a partially symptomatic treatment for amyloid-independent downregulation of ACh and a partially disease-modifying treatment for amyloid-dependent downregulation of ACh. We also consider that ChEIs such as donepezil have the ability to treat delirium.
Moreover, we propose measuring SAA in out-of-hospital pharmacies in order to reduce the patients' anticholinergic burden.

\section{Acknowledgments}

Funding for this study was received from Eisai Co. Ltd., Daiichi Sankyo Inc. and Ono Pharmaceutical Co. Ltd.

\section{Disclosure Statement}

Koji Hori received lecture fees from Eisai Co. Ltd., Pfizer Japan Inc., Novartis Pharma KK, Daiichi Sankyo Inc., Ono Pharmaceutical Co. Ltd., Janssen Pharmaceutical KK, Yoshitomi Yakuhin Co. and Mitsubishi Tanabe Pharma Co. Mitsugu Hachisu received funding from Astellas Pharma Inc., Meiji Seika Pharma Co. Ltd., Dainippon Sumitomo Pharm Co. Ltd., Eli Lilly Japan KK and Shionogi \& Co. Ltd., and received lecture fees from Meiji Seika Pharma Co. Ltd. and Mitsubishi Tanabe Pharma Co.
References
1 Whitehouse PJ, Price DL, Struble RG, Clark AW, Coyle JT, Delon MR: Alzheimer's disease and senile dementia: loss of neurons in the basal forebrain. Science 1982;215:12371239.

2 Butterfield DA, Pocernich CB: The glutamatergic system and Alzheimer's disease: therapeutic implications. CNS Drugs 2003; 17:641652.
3 Mabley JG, Pacher P, Szabo C: Activation of the cholinergic anti-inflammatory pathway reduces ricin-induced mortality and organ failure in mice. Mol Med 2009;15:166-172.

-4 Vandame D, Ulmann L, Teigell M, Prieto-Cappellini M, Vignon J, Privat A, Perez-Polo R, Nesic $\mathrm{O}$, Hirbec H: Development of NMDAR antagonists with reduced neurotoxic side effects: a study on GK11. PLoS One 2013;8:e81004. 
5 Nazarov PG, Krylova IB, Evdokimova NR, Nezhinskaya GI, Butyugov AA: C-reactive protein: a pentraxin with anti-acetylcholine activity. Life Sci 2007;80:2337-2341.

6 Hori K, Konishi K, Tomioka H, Tani M, Minegishi G, Tanaka H, Akita R, Yokoyama S, Oshio T, Hachisu M: Serum anticholinergic activity: a biomarker for rapid progression of Alzheimer's disease. J Autacoids 2012, DOI: org/10.4172/2161-0479.S4-001.

7 Perry EK, Kilford L, Lees AJ, Burn DJ, Perry $\mathrm{RH}$ : Increased Alzheimer pathology in Parkinson's disease related to antimuscarinic drugs. Ann Neurol 2003;54:235-238.

$>8$ Hori K, Konishi K, Tani M, Akashi N, Kitajima Y, Hachisu M: Anti-dementia agents are partially symptomatic treatment and partially disease modifying treatment. Brain Disord Ther 2014;3:6.

>9 Hori K, Konishi K, Akita R, Tani M, Tomioka H, Kitajima Y, Yokoyama S, Azuma K, Ikuse D, Akashi N, Yuda H, Hachisu M: Proposal of endogenous anticholinergic hypothesis in Alzheimer's disease. Jpn J Neuropsychopharmacol 2013;33,117-126.

10 Hori K, Konishi K, Tani M, Tomioka H, Akita R, Kitajima Y, Aoki M, Yokoyama S, Azuma K, Ikuse D, Akashi N, Hosoi M, Jinbo K, Hachisu M: Serum anticholinergic activity: a possible peripheral marker of the anticholinergic burden in the central nervous system in Alzheimer's disease. Dis Markers 2014;2014: 459013.
11 Hori K, Konishi K, Tani M, Tomioka H, Akita R, Kitajima Y, Aoki M, Kikuchi N, Ikuse D, Akashi N, Hosoi M, Jinbo K, Hachisu M: Why does the progression of Alzheimer's disease accelerate? Ann Psychiatry Ment Health 2014;2:1006.

12 Perry EK, Marshall E, Kerwin J, Smith CJ, Jabeen S, Cheng AV, Perry RH: Evidence of a monoaminergic-cholinergic imbalance related to visual hallucinations in Lewy body dementia. J Neurochem 1990;55:1454-1456.

13 McKeith IG, Dickson DW, Lowe J, Emre M, O’Brien JT, Feldman H, Cummings J, Duda JE, Lippa C, Perry EK, Aarsland D, Arai H, Ballard CG, Boeve B, Burn DJ, Costa D, Del Ser T, Dubois B, Galasko D, Gauthier S, Goetz CG, Gomez-Tortosa E, Halliday G, Hansen LA, Hardy J, Iwatsubo T, Kalaria RN, Kaufer D, Kenny RA, Korczyn A, Kosaka K, Lee VM, Lees A, Litvan I, Londos E, Lopez OL, Minoshima S, Mizuno Y, Molina JA, Mukaetova-Ladinska EB, Pasquier F, Perry RH, Schulz JB, Trojanowski JQ, Yamada M; Consortium on DLB: Diagnosis and management of dementia with Lewy bodies: third report of the DLB Consortium. Neurology 2005;65: 1863-1872.

14 Yilmaz MS, Işık B, Ongar M, Kavalci C, Solakoglu AG, Baba M, Kavalci G, Salt O: Delirium due to Datura stramoniun ingestion: a case report. Adv Res 2014;2:523-527.

-15 Hori K, Tominaga I, Inada T, Oda T, Hirai S, Hori I, Onaya M, Teramoto H: Donepezil-responsive alcohol-related prolonged delirium. Psychiatry Clin Neurosci 2003;57:603-604.
6 Konishi K, Hori K, Tomioka H, Minegishi G, Tani M, Tanaka H, Akita R, Yokoyama S, Oshio T, Hachisu M: Donepezil abolishes anticholinergic activity in a patient with amnesia. Pharmacology 2013;91:86-91.

17 Tune L, Carr S, Hoag E, Cooper T: Anticholinergic effects of drugs commonly prescribed for the elderly: potential means for assessing risk of delirium. Am J Psychiatry 1992;149: 1393-1394.

18 Konishi K, Hori K, Uchida H, Watanabe K, Tominaga I, Kimura M, Hosoyamada M, Shibasaki T, Kataoka A, Hachisu M: Adverse effects of anticholinergic activity on cognitive functions in Alzheimer's disease. Psychogeriatrics 2010;10:34-38.

19 Hori K, Konishi K, Watanabe K, Uchida H, Tsuboi T, Moriyasu M, Tominaga I, Hachisu $\mathrm{M}$ : Influence of anti-cholinergic activity in serum on clinical symptoms of Alzheimer's disease. Neuropsychobiology 2011;63:147-153.

20 Tune L, Coyle JT: Serum levels of anti-cholinergic drugs in treatment of acute extrapyramidal side effects. Arch Gen Psychiatry 1980;37: 293-297.

21 Hori K, Funaba Y, Konishi K, Moriyasu M, Hirata K, Oyamada R, Tominaga I, Inada T: Assessment of pharmacological toxicity using serum anticholinergic activity in a patient with dementia. Psychiatry Clin Neurosci 2005;59:508-510.
Pharmacotherapy for Neurocognitive Disorders Based on AA
Neurodegener Dis 2015;15:157-161 